\title{
CHARGE syndrome associated with de novo (I1460Rfs"15) frameshift mutation of CHD7 gene in a patient with arteria lusoria and horseshoe kidney
}

\author{
CRISTINA GUG $^{1 *}$, EUSEBIU VLAD GORDUZA ${ }^{2,3^{*}}$, ADRIAN LĂCĂTUŞU ${ }^{4}$, MONICA ADRIANA VAIDA $^{5}$ \\ FLORIN BÎRSĂŞTEANU ${ }^{6}$, MARIA PUIU ${ }^{1}$ and DORINA STOICĂNESCU ${ }^{1}$ \\ ${ }^{1}$ Department of Microscopic Morphology, Victor Babeş University of Medicine and Pharmacy, 300041 Timisoara; \\ ${ }^{2}$ Prenatal Diagnosis Department, Cuza Voda Obstetrics-Gynecology Clinical Hospital, 700038 Iasi; \\ ${ }^{3}$ Department of Medical Genetics, Grigore T. Popa University of Medicine and Pharmacy, 700115 Iasi; \\ Departments of ${ }^{4}$ Pediatrics, ${ }^{5}$ Anatomy and Embryology and ${ }^{6}$ Radiology and Medical Imaging, \\ Victor Babeş University of Medicine and Pharmacy, 300041 Timisoara, Romania
}

Received September 11, 2019; Accepted March 2, 2020

DOI: $10.3892 /$ etm.2020.8683

\begin{abstract}
CHARGE syndrome is an autosomal dominant condition caused by mutations in the chromodomain helicase DNA binding protein 7 (CHD7) gene. The present study reported on the case of a 16-month-old female with plurimalformative syndrome, whose etiology was identified by clinical whole-exome sequencing (WES) analysis. Clinical and follow-up assessments identified multiple craniofacial dysmorphisms, congenital defects and functional symptoms, including dysphagia and Marcus Gunn jaw winking synkinesis. Trio-WES analysis was performed for the patient and their parents and the presence of CHARGE syndrome was further indicated using single-molecule real-time sequencing. A de novo pathogenic variant, c.4379_4380del (p. Ile1460Argfs*15), was identified in exon 19 of the CHD7 gene, which resulted in a premature translational stop signal. Trio-WES analysis was used for further investigation, indicating that neither of the patient's parents had the mutation and confirming its de novo nature. To the best of our knowledge, the case of the present study was the first reported case of CHARGE syndrome in Romania with congenital
\end{abstract}

Correspondence to: Dr Adrian Lăcătuşu, Department of Pediatrics, Victor Babeş University of Medicine and Pharmacy, P-ta E. Murgu 2, 300041 Timisoara, Romania

E-mail: adilacatusu@yahoo.com

Dr Monica Adriana Vaida, Department of Anatomy and Embryology, Victor Babeş University of Medicine and Pharmacy, P-ta E. Murgu 2, 300041 Timisoara, Romania

E-mail: monicaadrianavaida@yahoo.com

*Contributed equally

Key words: CHARGE syndrome, CHD7 gene, frameshift mutation, arteria lusoria, horseshoe kidney defects including an aberrant right subclavian artery and a horseshoe kidney. CHARGE syndrome was diagnosed in the patient based on the pathogenic mutation in the CHD7 gene. To the best of our knowledge, the present case report is the first to suggest that the CHD7 gene variant is associated with CHARGE syndrome.

\section{Introduction}

CHARGE syndrome is a rare, autosomal dominant disorder characterized by multiple congenital anomalies. According to Orphanet, CHARGE syndrome Online Mendelian Inheritance of Man entry no. 214800; https://www.orpha. net/consor/cgi-bin/) has a prevalence of 1-9/100,000. CHARGE is an acronym for the most common symptoms observed in patients with the condition: Coloboma, heart defects, atresia of the choanae, retarded growth and/or development, genital hypoplasia and ear abnormalities (1). However, other clinical manifestations have also been identified in diagnosed cases. Patients with CHARGE syndrome exhibit complex symptoms that differ from one case to another, indicating the variable clinical expression of the disorder. Cranial nerve (CN) dysfunction, external ear anomalies, semicircular canal anomalies, coloboma, choanal atresia, genital hypoplasia, congenital cardiovascular defects, cleft lip and/or palate and anosmia are identified in $>50 \%$ of patients. Motor retardation, swallowing and severe feeding problems, and intellectual disability are also frequent among patients with CHARGE syndrome (2).

The identification of clinical features is important for the diagnosis of patients with suspected CHARGE syndrome (3). Sanlaville and Verloes (4) divided the clinical aspects of CHARGE syndrome into major and minor criteria, but the highly variable clinical expression and the lack of obligatory elements have continued to pose major diagnostic problems. Major and minor clinical criteria have been refined and updated (4,5), and a new checklist organized by body system and age has been developed to aid the diagnosis of CHARGE syndrome (6). Recently, a novel genetic approach has changed 
the importance of clinical criteria for the diagnosis of the disorder. Hale et al (7) suggested that difficulties regarding the clinical diagnosis of CHARGE syndrome may be overcome by including the pathogenic chromodomain helicase DNA binding protein 7 (CHD7) variant as a major diagnostic criterion. Accordingly, a pathogenic CHD7 variant status plus one major feature would be sufficient for the diagnosis of the disorder $(7,8)$. It is important for each novel mutation that extends the spectrum of pathogenic mutations in the CHD7 gene to be included in the databases, to facilitate the diagnosis and estimate the prevalence of the disorder (3). Since the publication of the new criteria in 2016, only a few case reports discussing patients with CHARGE syndrome with a pathogenic mutation in the CHD7 gene, previously considered as atypical CHARGE, have been published $(9,10)$. By understanding the different genotypes of CHARGE syndrome, it was possible to make clear genotype-phenotype associations for all CHD7 variants.

In the present study, a case with a plurimalformative syndrome, whose etiology was identified by clinical whole-exome-sequencing (WES) analysis, was described. According to the criteria of Hale et al (7), the patient was diagnosed with CHARGE syndrome due to the presence of the pathogenic CHD7 variant. By reporting a novel case of CHARGE syndrome, the present study emphasizes the importance of the new criteria, which may encourage clinicians to perform extensive genetic testing, e.g., WES, for an accurate diagnosis of the disorder.

\section{Materials and methods}

Clinical and paraclinical evaluation. Physical examination of the patient was followed by: Ear, nose and throat assessment, audiogram, thoracic computed tomography (CT) and abdominal and heart Doppler ultrasonography.

Genetic analysis. Genomic DNA was extracted from peripheral venous blood, as previously described by Miller et al (11). Clinical WES was used to detect an exonic pathogenic variant that may explain the phenotype of the patient. Gene sequencing was performed by the Invitae Laboratory. Genomic DNA was enriched for targeted regions using a hybridization-based protocol and sequenced using Illumina technology (Illumina, Inc.). The average coverage depth of the WES of all targeted regions was $\geq 50 \mathrm{X}$. Reads were aligned to a reference sequence Genome Reference Consortium human build 37 (GRCh37) (12) and sequence changes were identified and interpreted in the context of a single clinically relevant transcript. Exonic deletions and duplications were identified using an algorithm that determined the copy number at each target. Subsequently, the biological and clinical implications of each variant were identified. All reported pathogenic or likely pathogenic variants that had potential medical implications for the patient were verified by orthogonal technologies. Single molecule real-time (SMRT) sequencing technology (Pacific Biosciences, Inc.) was used to further verify the results of the WES analysis. Using a combination of public tools and databases, a comprehensive search of phenotype-genotype associations was performed. Disruptive variants, including variants that caused premature truncation events, interfered with canonical splice sites or resulted in initiator, frameshift or multi-exon deletions that were detected in genes associated with Mendelian conditions that corresponded to the patient's presentation, were reported.

Subsequently, trio-WES analysis was performed on samples obtained from the parents of the patient, which identified the mutation as a de novo variant that was only present in the patient.

The variants that were identified as potentially explaining the clinical features of the patient were reported according to the guidelines established by the American College of Genetics and Genomics (13). Sequence variants with a minor allele frequency of $>0.05$ in the Human Genetic Variation Database (http://www.genome.med.kyoto-u.ac.jp/SnpDB/) and the NHLBI Grand Opportunity Exome Sequencing Project (http://evs. gs.washington.edu/EVS/) were excluded.

\section{Results}

Case presentation. The present study described the case of a 16-month-old female who was evaluated in the Louis T,urcanu Emergency Hospital for Children and in the Pediatric Cardiology Department of Pius Brînzeu Emergency County Clinical Hospital, Timisoara, Romania, in February 2019, for dysmorphic features and multiple congenital defects in order to establish a diagnosis and to aid in the management of a future pregnancy. The patient was born post-term via normal spontaneous delivery. The parents (mother, 33 years; father, 34 years) were non-consanguineous and appeared healthy. The patient had no siblings. Ultrasonography performed during the pregnancy did not detect any pathological changes in the patient. Anthropometric measurements at birth were within the normal ranges: Body weight, 3,630 g; body height, $52 \mathrm{~cm}$; and cranial perimeter, $35 \mathrm{~cm}$. The Apgar scores of the patient were 5/6/10 and the family history of the patient was unremarkable.

Medical history. The patient displayed facial dysmorphism from birth, which is the reason for further investigation performed by the neonatology and pediatric services. At birth, severe dysphagia was reported; therefore, enteral nutrition was the recommended feeding route. The cause of dysphagia was investigated and neuromuscular dysphagia, tumors and hemangioma were excluded as causes of the condition. In time, the patient's condition improved and at 3 months of age, the patient was fed orally. Thoracic and abdominal CT scans were performed, revealing an aberrant right subclavian artery (arteria lusoria) and a horseshoe kidney. The patient was not receiving any medication at the time of presentation.

Clinical evaluation. At 16 months of age, the anthropometric measurements of the patient upon first physical examination in the Genetics Department were as follows: Body height, $75 \mathrm{~cm}$; body weight, 8,730 $\mathrm{g}$; and cranial perimeter, $45.5 \mathrm{~cm}$ (in the normal range) (14). Upon physical examination at 21 months of age, the following anthropometric measurements were recorded: Body height, $78 \mathrm{~cm}$; body weight, $10 \mathrm{~kg}$ (in the normal range); and cranial perimeter, $46.6 \mathrm{~cm}$ (in the normal range) (14). At 16 and 21 months of age, the patient was fed only with liquids and semisolids. 

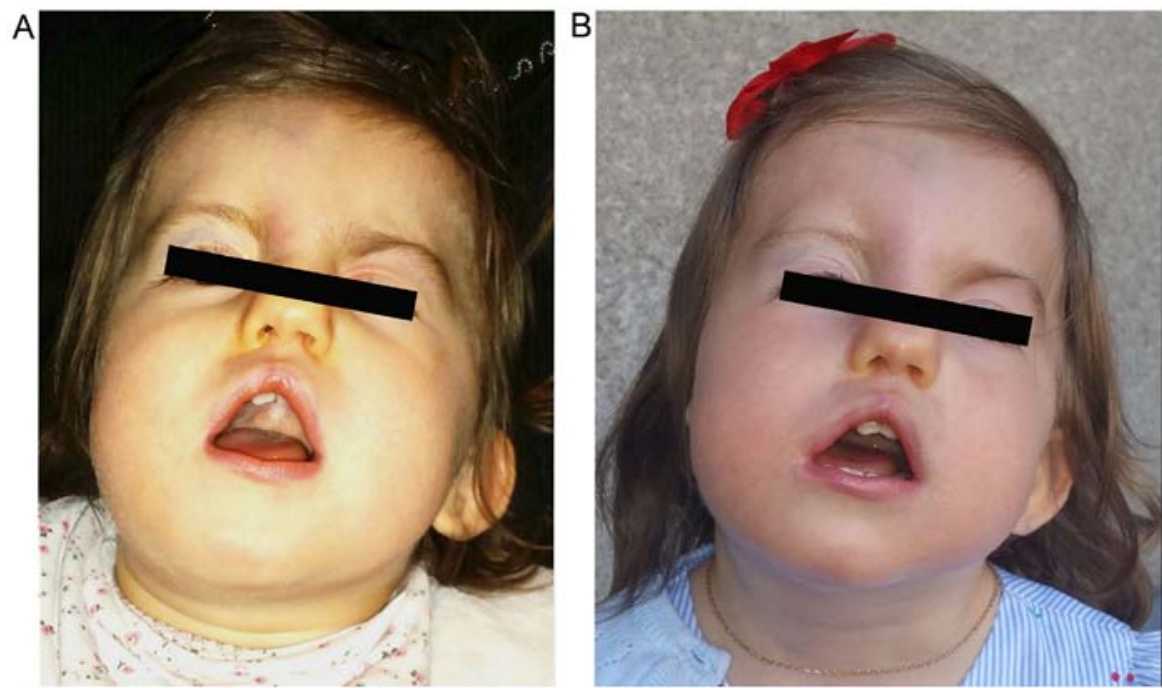

Figure 1. Facial dysmorphy of the patient included craniofacial asymmetry, square face, prominent forehead, high palate, triangular-shaped open mouth, asymmetric and low-set left ear, left palpebral ptosis, left facial palsy and head retroflexion. Facial images at (A) 16 months and (B) 21 months of age.
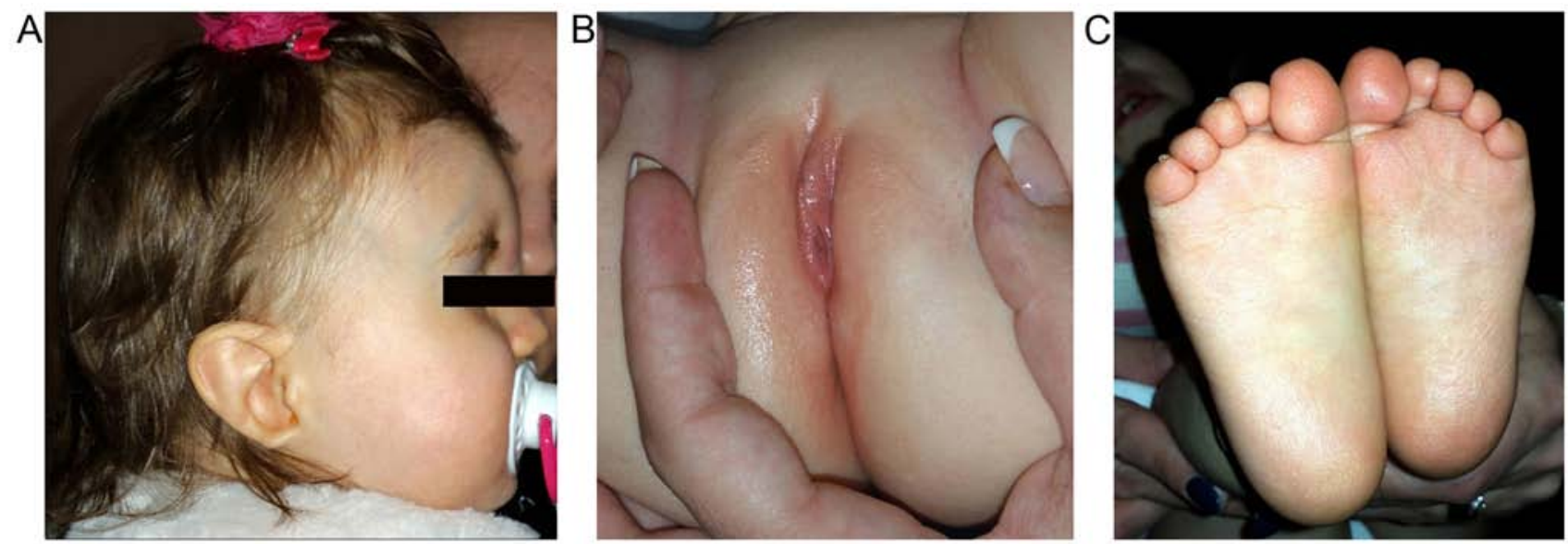

Figure 2. (A) Patient's profile: Low-set left ear, ear lobe agenesis on the right side, (B) labia minora hypoplasia, (C) soles without furrows, but with fine sole dermatoglyphics.

The patient displayed dysmorphic facial features, which included square face, craniofacial asymmetry, prominent forehead, wide nasal ridge, high palate, triangular-shaped open mouth, low-set left ear, asymmetry of the shape and size of the ears, abnormality of the pinna cartilage of the outer ear, ear lobe agenesis on the right side and unilateral left blepharoptosis with associated upper eyelid contraction (Figs. 1 and 2). Ophthalmic examination revealed choroidal coloboma.

The patient also presented with fixed head retroflexion, congenital craniofacial dysostosis, facial palsy (left hemi-face), general facial hypotonia, facial grimacing, abnormal conjugate eye movement and Marcus Gunn jaw winking synkinesis. The aforementioned symptoms, together with the left blepharoptosis, conferred a peculiar facial expression. Cranial nerves (CN) VII, VIII, IX and X were affected. The patient suffered from profound deafness.

Further anomalies that were identified by systemic examination included labia minora hypoplasia, abnormality of the plantar skin and abnormal plantar dermatoglyphics (Fig. 2). In addition, the patient was only able to walk with support.
Nasal endoscopic exam revealed that the patient did not have choanal atresia, while audiogram revealed severe bilateral hearing impairment. The patient did not speak, but she smiled and was able to cooperate. The left auditory nerve was not visualized on cranial CT.

Thoracic CT identified an aberrant origin of the right subclavian artery (arteria lusoria) without obstructive manifestations of the esophagus or trachea (Fig. 3).

Heart ultrasonography revealed that the patient did not display any intracardiac structural abnormalities; therefore, the only cardiac defect identified that was associated with CHARGE syndrome was the aberrant origin of the right subclavian artery (Fig. 4).

The patient did not receive any medication. After receiving kinesiotherapy for $2 \mathrm{~h}$ daily, the patient was able to maintain equilibrium on the feet and walk short distances without support and retroflexion of the head and left palpebral ptosis were reduced. Cardiology follow-up for evaluation of the cardiac status was recommended. A cochlear implant was scheduled. 

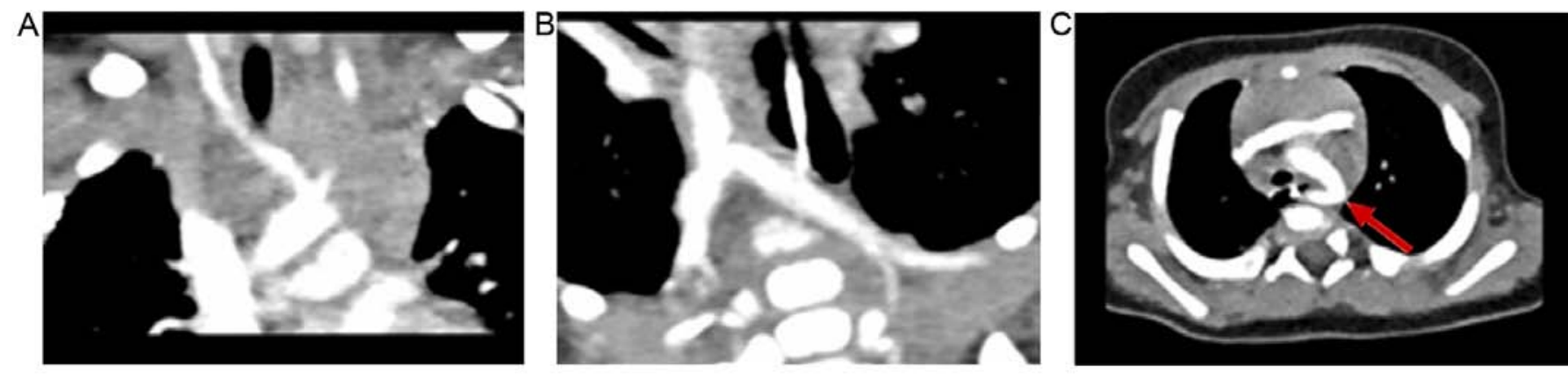

Figure 3. Computed tomograms displaying (A) the right common carotid artery and left common carotid artery, both arising from the aortic arch; (B) the left subclavian artery and right subclavian artery with aberrant origin and diverticulum of Kommerell (the distal remnant of the right aortic arch), and (C) the left-sided aortic arch with an aberrant right subclavian artery arising from the proximal part of the descending aorta, with retrotracheal and retro-oesophageal course, but without obstruction. The arrow indicates the right subclavian artery emerging from proximal descending aorta.
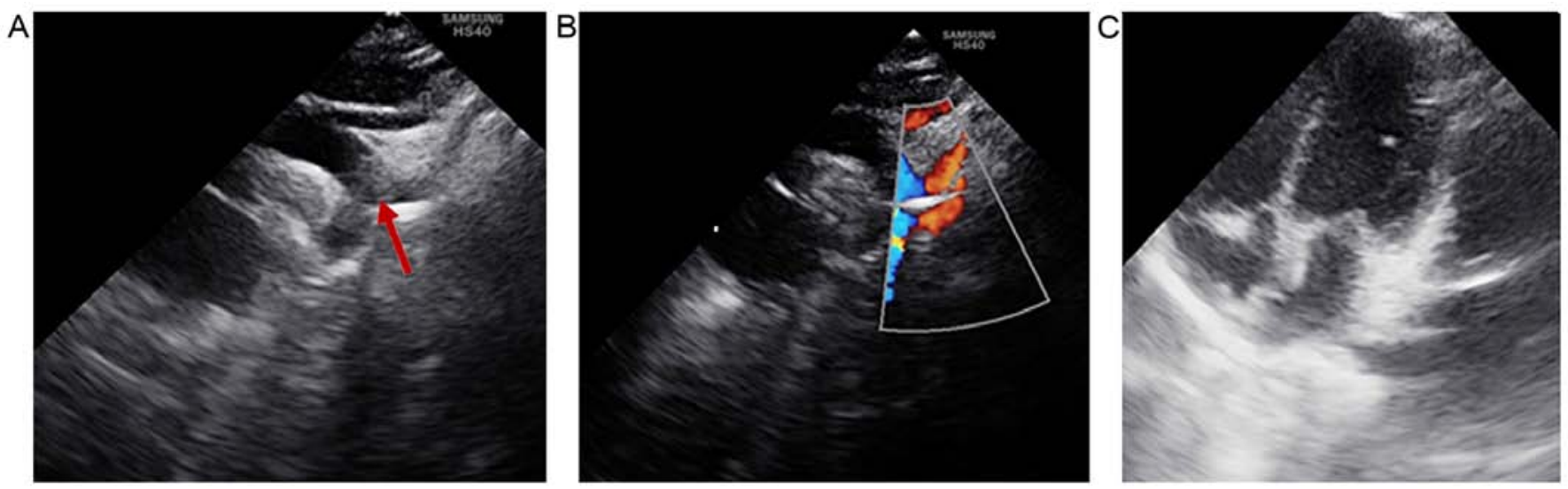

Figure 4. Heart ultrasonography. (A) The suprasternal window displays the left-sided aortic arch with the aberrant origin of the right subclavian artery (arrow), which arises from the proximal part of the descending aorta. (B) The suprasternal window with color Doppler flow displays the left-sided aortic arch with flow across the aberrant right subclavian artery (arrow), right common carotid artery and left subclavian artery. (C) Apical four-chamber window displaying normal cardiac structures.

Mutation. A de novo pathogenic variant, c.4379_4380del (p.Ile1460Argfs"15), was identified in exon 19 of the CHD7 gene. The variant consisted of one thymine deletion from position 4,379 and one adenine deletion from position 4,380 (rs398124319). The mutation led to a reading frameshift starting from isoleucine at codon 1,460 , which resulted in a premature translational stop signal (p.Ile1460Argfs*15) and was hypothesized to result in an absent or disrupted protein product. The analysis identified a monoallelic deletion in the CHD7 gene, NM_017780.3 (CHD7, c.4379_4380del), which was also identified by SMRT sequencing (Fig. 5).

The mutation was present in the Human Gene Mutation Database (http://www.hgmd.org) and the Clinical Variance database (http://www.ncbi.nlm.nih.gov/clinvar). The heterozygous frameshift mutation was not detected in the parents of the patient, suggesting that it was a de novo mutation.

\section{Discussion}

CHARGE syndrome is a variable disorder that affects a number of different parts of the body and is characterized by a non-random cluster of congenital anomalies and dysmorphisms, with significant variation between patients (15). The diagnosis relies on a set of major and minor clinical criteria $(4,5)$. According to the criteria of Sanlaville and Verloes (4), a patient must display the following: Three major criteria, or two major and two minor criteria for diagnosis with the typical form; two major and one minor criterion for diagnosis with the partial form; two major but no minor criterion, or one major and two minor criteria for diagnosis with the atypical form (4). Hale et al (7), proposed an update to the clinical diagnostic criteria, suggesting that a pathogenic CHD7 variant status should be considered as a major diagnostic criterion, which, when present together with one other major criterion, is sufficient for the diagnosis of typical CHARGE syndrome.

The majority of patients with CHARGE syndrome display distinctive facial features, including a square-shaped face and facial asymmetry, which were also observed in the case described in the present study (3). Patients with CHARGE syndrome also display ear abnormalities, which may contribute to hearing problems. The case reported in the present study displayed abnormalities of the shape and position of the bilateral ears, as well as ear lobe agenesis on the right side.

The central nervous system is also frequently affected in patients with CHARGE syndrome, with $\mathrm{CN}$ dysfunction reported in $>90 \%$ of cases. Different clinical manifestations occur when $>1 \mathrm{CN}$ is dysfunctional. In the case reported in the 


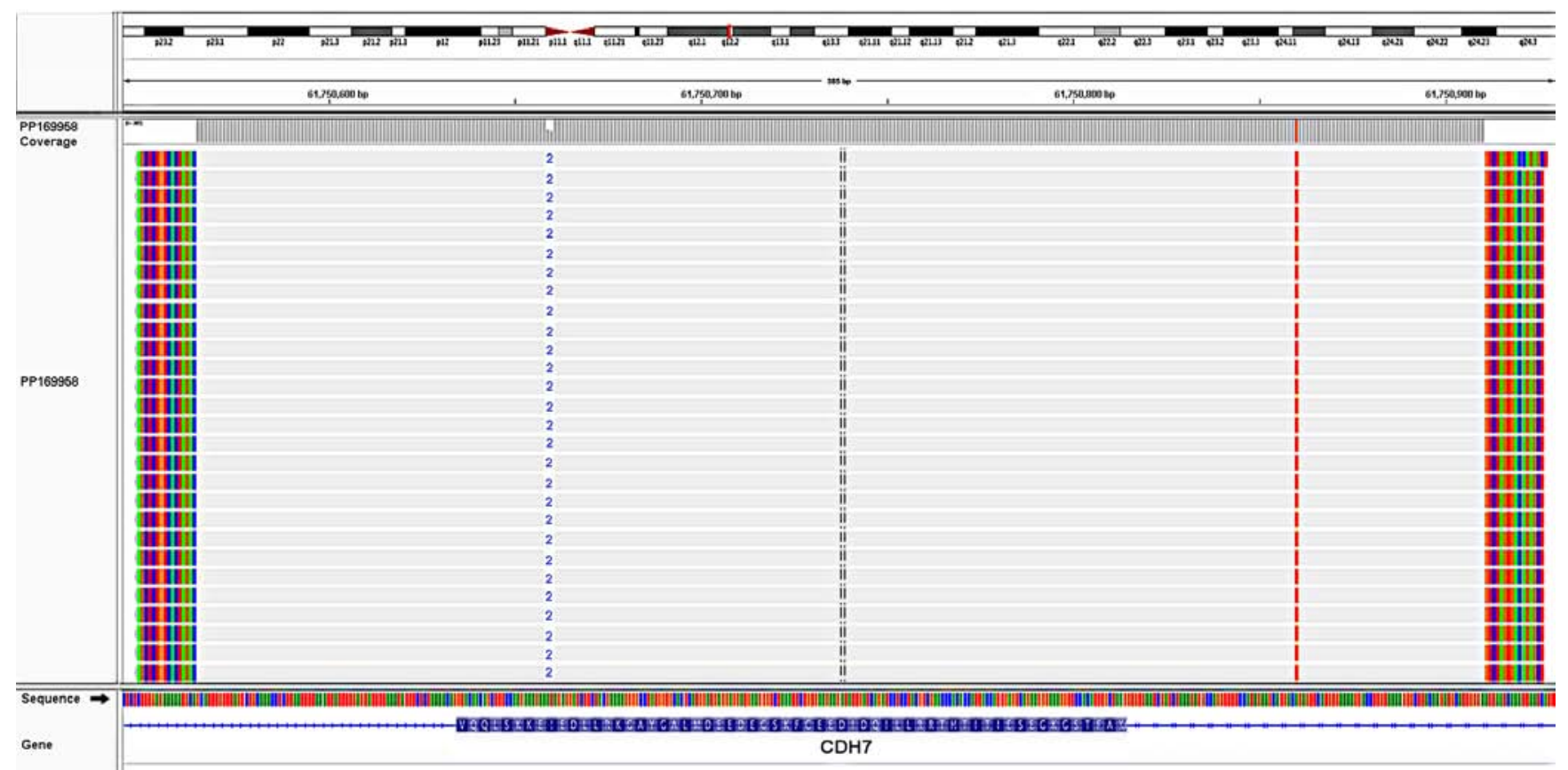

Figure 5. Pacific Bioscience long reads identify the heterozygous 2-bp deletion causing a frameshift, which started at codon 1,460 of the CHD7 gene (p.Ile1460Argfs"15). CHD7, chromodomain helicase DNA binding protein 7.

present study, CN VII, VIII, IX and X were affected. The patient suffered from profound deafness and the cranial CT indicated that the patient had no left auditory nerve. Of note, visual and hearing impairments, as well as intellectual disability, are frequently observed in patients with CHARGE syndrome (16). Unilateral left facial palsy was first reported at birth in the patient; however, at the time of the present study, it had not been determined whether the patient had a full or diminished sense of smell, or complete anosmia, coordinated by CN I.

The minor characteristics of CHARGE syndrome include slow growth starting in late infancy, delayed sitting and unsupported walking, all of which were present in the case reported in the present study. The patient also displayed congenital cardiovascular defects, including an aberrant origin of the right subclavian artery (arteria lusoria), a horseshoe kidney, VII and VIII CN palsy and coloboma, which is a major criterion. The presence of one major and three minor criteria suggested that the patient had atypical CHARGE syndrome, according to the Sanlaville and Verloes criteria (3).

Congenital heart defects are present in 3/4 of patients with CHARGE syndrome (17). The types of congenital heart defect are variable, from conotruncal and atrioventricular septal defects to aortic arch anomalies. Among the congenital aortic arch anomalies, the aberrant origin of the right subclavian artery is the most common (18).

The patient presented with severe dysphagia immediately after birth with unknown causes, which spontaneously remitted after several months. The thoracic CT displayed the aberrant right subclavian artery, which may have been the cause of dysphagia. However, at the age of 16 months, the patient did not have any dysphagia; therefore, surgical intervention was not required and only cardiac monitoring was recommended.

A horseshoe kidney without impaired renal function was identified in the patient. Urinary system anomalies are present in $10-40 \%$ of patients with CHARGE syndrome (19). Other minor abnormalities, such as hypoplastic labia minora, also cause differential diagnostic problems with Meier-Gorlin syndrome type 1 or 3, Prader-Willi syndrome, Schinzel-Giedion syndrome, mixed gonadal dysgenesis $(20,21)$. Several clinical features of these syndromes overlap with CHARGE syndrome, but they also have distinctive signs. All these syndromes were excluded based on clinical findings and on the presence of pathogenic mutation in the CHD7 gene found in the patient in the present case report.

The patient received follow-up assessments for 6 months and the clinical re-evaluation performed after 6 months revealed an important improvement. A skeletal survey and a dual-energy X-ray absorptiometry scan for osteoporosis were recommended for the patient $(5,22)$. The patient cooperated, smiled and responded to non-verbal stimuli. By investigating the range of developmental abilities observed in patients with CHARGE syndrome, as measured by the Adaptive Behavior Evaluation Scale, researchers concluded that the age at which the patient was able to walk was the best predictor of scores (23). The IQ of patients with CHARGE syndrome ranges from near-normal to severe retardation. The behavioral phenotype typically consists of low-level adaptive behavioral skills (e.g., social skills, seeking of attention from others, externalizing problems) and symptoms of autism-like spectrum disorder. Data regarding a unique behavioral phenotype for patients with CHARGE syndrome are emerging (5).

The CHD7 gene is associated with autosomal-dominant CHARGE syndrome (24). Regarding genotype-phenotype correlations, missense mutations are associated with milder phenotypes and variants leading to a premature stop codon are associated with more severe phenotypes $(2,25,26)$. The case described in the present study displayed a severe 
phenotype and a pathogenic variant (c.4379_4380del) was identified. The mutation resulted in a premature translational stop signal (p.Ile1460Argfs*15) in exon 19 of the CHD7 gene. Several studies have reported that frameshift mutations are the most common type of mutation in patients with CHARGE syndrome, with a frequency of $34-73 \%(8,27,28)$. Clinical WES analysis takes into account information regarding the target genes associated with the major symptoms, as well as the verification of the genes indicated by the American College of Medical Genetics (13,29,30). Compared to classical sequencing, WES analysis has a shorter analysis time, lower costs and offers a more precise diagnosis. In the present study, Trio-WES analysis was performed, which allowed the de novo mutation to be identified, as neither of the patient's parents displayed the mutation in the $\mathrm{CDH} 7$ gene. In a large study, parental DNA profiles were assessed and all pathogenic variants were identified as de novo mutations, except for one case (8). An intragenic deletion was identified in two brothers, but was not identified in the blood samples of the parents. It was hypothesized that one parent had germinal mosaicism (8). Considering this possibility, prenatal diagnosis should be recommended for each future pregnancy $(31,32)$. De novo mutations are more frequent than inherited mutations and the majority are frameshift mutations (33-35). Although rare, pathogenic variants may be inherited from a mildly affected parent; therefore, parents should be carefully examined $(3,4)$. An analysis of a French cohort suggested that $66.38 \%$ of patients with CHARGE syndrome had different pathogenic variants and $90 \%$ of these variants were novel (8). It is estimated that $74 \%$ of patients with CHARGE syndrome have congenital heart defects due to a CHD7 mutation (25). Congenital heart defects are more frequent in patients with truncating variants of the gene $(80 \%)$ compared with patients with missense or splice-site variants (58\%) (25). Aortic arch anomalies, frequently including an aberrant subclavian artery or right aortic arch, were reported in $14 \%$ of cases in a previous study (18). In the present study, the patient was the first case of reported CHARGE syndrome in Romania presenting with a truncating CHD7 mutation and an aberrant subclavian right artery. Another case with an unspecified mutation in the CHD7 gene has also been reported in Romania (36).

The variant identified in the present study, c.4379_4380del in the CHD7 gene, was not present in population databases (Exome Aggregation Consortium: No frequency) and appeared to have not been reported in the literature. ClinVar contains an entry for this variant (variation ID: 95789) that is considered to be a pathogenic and germinal mutation. Over 500 mutations in the CHD7 gene have been reported (28), but only one case had the mutation (https://www.ncbi.nlm. nih.gov/clinvar/variation/95789/) that was identified in the present study and it has not been reported in a published scientific journal associated with CHARGE syndrome. The rare variant c.4379_4380del in the CHD7 gene is consistent with a loss-of-function variant, which is known to be pathogenic (37), and haploinsufficiency is the most likely pathogenic mechanism (38).

CHD7 mutations cause inactivation of remodeling activity of varying degrees. Remodeling of nucleosomes is a key function of CHD7 during developmental processes, which may explain why CHD7 mutations cause developmental defects but do not prevent the development of pregnancy (39-42).

To the best of our knowledge, the present study reported the first case of CHARGE syndrome in Romania that displayed an aberrant right subclavian artery and a horseshoe kidney, and was clearly diagnosed on the basis of the pathogenic mutation in the $\mathrm{CDH} 7$ gene. The frameshift mutation resulted in the formation of a premature stop codon leading to the inactivation of the CHD7 protein. CHARGE syndrome is a rare disease; therefore, every newly reported case improves the understanding of the spectrum of mutations in the $\mathrm{CDH} 7$ gene and extends the knowledge of genotype-phenotype correlations.

\section{Acknowledgements}

Not applicable.

\section{Funding}

No funding was received.

\section{Availability of data and materials}

The datasets used and/or analyzed during the present study are available from the corresponding author on reasonable request.

\section{Authors' contributions}

CG and DS contributed to the conceptualization of the study, literature search as well as writing, editing and critically reviewing the manuscript; $\mathrm{CG}, \mathrm{AL}$ and $\mathrm{FB}$ performed data analysis; EVG and MAV performed data analysis, critical review and supervision. MP treated the patient in the clinic throughout the admission and provided patient data. All authors have read and approved the final manuscript for publication.

\section{Ethics approval and consent to participate}

The study described in this article was carried out in accordance with the Code of Ethics of the World Medical Association (Declaration of Helsinki) for experiments involving humans and Uniform Requirements for manuscripts submitted to biomedical journals. The study was approved by the Local Ethics Commission for Scientific Research of Pius Brînzeu Emergency County Clinical Hospital Timisoara, Romania; (approval no. 172/05.08.2019). The parents of the patient provided written informed consent regarding genetic testing and participation in the study.

\section{Patient consent for publication}

The parents of the patient provided written informed consent regarding the publication of the medical data and images of the case.

\section{Competing interests}

The authors declare that they have no competing interests. 


\section{References}

1. Pagon RA, Graham JM Jr, Zonana J and Yong SL: Coloboma, congenital heart disease, and choanal atresia with multiple anomalies: CHARGE association. J Pediatr 99: 223-227, 1981.

2. Basson MA and van Ravenswaaij-Arts C: Functional insights into chromatin remodelling from studies on CHARGE syndrome. Trends Genet 31: 600-611, 2015.

3. Hsu P, Ma A, Wilson M, Williams G, Curotta J, Munns CF and Mehr S: CHARGE syndrome: A review. J Paediatr Child Health 50: 504-511, 2014.

4. Sanlaville D and Verloes A: CHARGE syndrome: An update. Eur J Hum Genet 15: 389-399, 2007.

5. Blake KD and Prasad C: CHARGE syndrome. Orphanet J Rare Dis 1: 34, 2006.

6. Trider CL, Arra-Robar A, van Ravenswaaij-Arts C and Blake K Developing a CHARGE syndrome checklist: Health supervision across the lifespan (from head to toe). Am J Med Genet A 173: 684-691, 2017.

7. Hale CL, Niederriter AN, Green GE and Martin DM: Atypical phenotypes associated with pathogenic CHD7 variants and a proposal for broadening CHARGE syndrome clinical diagnostic criteria. Am J Med Genet A 170A: 344-354, 2016.

8. Legendre M, Abadie V, Attié-Bitach T, Philip N, Busa T, Bonneau D, Colin E, Dollfus H, Lacombe D, Toutain A, et al Phenotype and genotype analysis of a French cohort of 119 patients with CHARGE syndrome. Am J Med Genet C Semin Med Genet 175: 417-430, 2017

9. Pranckėnienė L, Preikšaitienė E, Gueneau L, Reymond A and Kučinskas V: De novo duplication in the CHD7 gene associated with severe CHARGE syndrome. Genomics Insights 12 1178631019839010,2019

10. Katoh-Fukui Y, Yatsuga S, Shima H, Hattori A, Nakamura A, Okamura K, Yanagi K, Iso M, Kaname T, Matsubara Y, et al: An unclassified variant of CHD7 activates a cryptic splice site in a patient with CHARGE syndrome. Hum Genome Var 5: 18006, 2018.

11. Miller SA, Dykes DD and Polesky HF: A simple salting out procedure for extracting DNA from human nucleated cells. Nucleic Acids Res 16: 1215, 1988.

12. https://www.ncbi.nlm.nih.gov/assembly/GCF_000001405.13/-. Accessed in July 15, 2019.

13. Richards S, Aziz N, Bale S, Bick D, Das S, Gastier-Foster J, Grody WW, Hegde M, Lyon E, Spector E, et al; ACMG Laboratory Quality Assurance Committee: Standards and guidelines for the interpretation of sequence variants: A joint consensus recommendation of the American College of Medical Genetics and Genomics and the Association for Molecular Pathology. Genet Med 17: 405-424, 2015.

14. Boia M, Iacob D, Manea A, Budisan C, Enătescu I, Dima M and Costescu O: Creșterea şi dezvoltarea postnatală. In: Noțiuni Practice de Puericultură. Babeş V (ed). Timisoara, pp32-43, 2019 (In Romanian)

15. Delahaye A, Sznajer Y, Lyonnet S, Elmaleh-Bergès $M$, Delpierre I, Audollent S, Wiener-Vacher S, Mansbach AL, Amiel J, Baumann C, et al: Familial CHARGE syndrome because of CHD7 mutation: Clinical intra- and interfamilial variability. Clin Genet 72: 112-121, 2007.

16. Hudson A, Trider CL and Blake K: CHARGE Syndrome. Pediatr Rev 38: 56-59, 2017

17. Pierpont ME, Brueckner M, Chung WK, Garg V, Lacro RV McGuire AL, Mital S, Priest JR, Pu WT, Roberts A, et al; American Heart Association Council on Cardiovascular Disease in the Young; Council on Cardiovascular and Stroke Nursing; and Council on Genomic and Precision Medicine: Genetic basis for congenital heart disease: Revisited: A scientific statement from the American Heart Association. Circulation 138: e653-e711, 2018.

18. Corsten-Janssen N, van Ravenswaaij-Arts CMA and Kapusta L: Congenital arch vessel anomalies in CHARGE syndrome: A frequent feature with risk for co-morbidity. Int J Cardiol Heart Vasc 12: 21-25, 2016.

19. Roider L, Abdelaziz A and Gaballah AH: CHARGE syndrome with high bifurcation of the abdominal aorta and a horseshoe kidney: A case report. J Vasc Interv Radiol 29: 1288-1290.e1, 2018.

20. Velea PI, Mogoi M, Dema A, David V, Gug C and Paul C: Mixed gonadal dysgenesis associated with short stature and gonadoblastoma. Case Rep Acta Endo Buc 11: 221-227, 2015.

21. https://www.ncbi.nlm.nih.gov/medgen/376558. Accessed in March 5, 2020
22. Cevei M, Cssepento C, Gasparik AI and Stoicanescu D: Utility of risk factors in the current diagnosis of osteoporosis. Osteoporos Int 26: 184, 2015.

23. Salem-Hartshorne $\mathrm{N}$ and Jacob S: Adaptive behavior in children with CHARGE syndrome. Am J Med Genet A 133A: 262-267, 2005.

24. https://www.ncbi.nlm.nih.gov/medgen/?term=75567-. Accessed in July $5,2019$.

25. Corsten-Janssen N, Kerstjens-Frederikse WS, du Marchie Sarvaas GJ, Baardman ME, Bakker MK, Bergman JE, Hove HD, Heimdal KR, Rustad CF, Hennekam RC, et al: The cardiac phenotype in patients with a CHD7 mutation. Circ Cardiovasc Genet 6: 248-254, 2013.

26. Bergman JE, Janssen N, van der Sloot AM, de Walle HE, Schoots J, Rendtorff ND, Tranebjaerg L, Hoefsloot LH, van Ravenswaaij-Arts CM and Hofstra RM: A novel classification system to predict the pathogenic effects of CHD7 missense variants in CHARGE syndrome. Hum Mutat 33: 1251-1260, 2012

27. Zentner GE, Layman WS, Martin DM and Scacheri PC: Molecular and phenotypic aspects of CHD7 mutation in CHARGE syndrome. Am J Med Genet A 152A: 674-686, 2010.

28. Janssen N, Bergman JE, Swertz MA, Tranebjaerg L, Lodahl M, Schoots J, Hofstra RM, van Ravenswaaij-Arts CM and Hoefsloot LH: Mutation update on the CHD7 gene involved in CHARGE syndrome. Hum Mutat 33: 1149-1160, 2012.

29. Gug C, Mihaescu A and Mozos I: Two mutations in the thiazide-sensitive $\mathrm{NaCl}$ co-transporter gene in a Romanian Gitelman syndrome patient: Case report. Ther Clin Risk Manag 14: 149-155, 2018

30. Gug C, Caba L, Mozos I, Stoian D, Atasie D, Gug M and Gorduza EV: Rare splicing mutation in COL1A1 gene identified by whole exomes sequencing in a patient with osteogenesis imperfecta type I followed by prenatal diagnosis: A case report and review of the literature. Gene 741: 144565; Epub ahead of print, 2020.

31. Pauli S, Pieper L, Häberle J, Grzmil P, Burfeind P, Steckel M, Lenz U and Michelmann HW: Proven germline mosaicism in a father of two children with CHARGE syndrome. Clin Genet 75: 473-479, 2009.

32. Gug C, Huțanu D, Vaida M, Doroş G, Popa C, Stroescu R, Furău G, Furău C, Grigorită L and Mozos I: De novo unbalanced translocation $\mathrm{t}(15 ; 22)(\mathrm{q} 26.2 ; \mathrm{q} 12)$ with velo-cardio-facial syndrome: A case report and review of the literature. Exp Ther Med 16: 3589-3595, 2018.

33. Jongmans MC, Admiraal RJ, van der Donk KP, Vissers LE, Baas AF, Kapusta L, van Hagen JM, Donnai D, de Ravel TJ, Veltman JA, et al: CHARGE syndrome: The phenotypic spectrum of mutations in the CHD7 gene. J Med Genet 43: 306-314, 2006

34. Martínez-Quintana E, Rodríguez-González F, Garay-Sánchez P and Tugores A: Novel frameshift CHD7 mutation related to CHARGE syndrome. Mol Syndromol 5: 36-40, 2014.

35. Xu YP, Shi LP and Zhu J: Atypical CHARGE associated with a novel frameshift mutation of CHD7 in a Chinese neonatal patient. BMC Pediatr 18: 203, 2018.

36. Boer MC, Musat G and Budisteanu M: CHARGE syndromea rare cause of nose and ear anomalies. A case report. Rom J Rhinol 3: 45-48, 2013

37. Marcos S, Sarfati J, Leroy C, Fouveaut C, Parent P, Metz C, Wolczynski S, Gérard M, Bieth E, Kurtz F,et al: The prevalence of CHD7 missense versus truncating mutations is higher in patients with Kallmann syndrome than in typical CHARGE patients. J Clin Endocrinol Metab 99: E2138-2143, 2014.

38. Kohmoto T, Shono M,Naruto T, Watanabe M,SugaK, Nakagawa R, Kagami S, Masuda K and Imoto I: A novel frameshift mutation of CHD7 in a Japanese patient with CHARGE syndrome. Hum Genome Var 3: 16004, 2016.

39. Jurca A, Kinga K, Bembea M, Gug C and Jurca C: Fanconi anemia with cleft palate. Rev Med Chir Soc Med Nat Iasi 118: 1074-1077, 2014

40. Bouazoune K and Kingston RE: Chromatin remodeling by the CHD7 protein is impaired by mutations that cause human developmental disorders. Proc Natl Acad Sci USA 109: 19238-19243, 2012.

41. Gug C, Rațiu A, Navolan D, Drăgan I, Groza IM, Păpurică M, Vaida MA, Mozos I and Jurcă MC: The incidence and spectrum of chromosomal anomalies in miscarriage samples: A retrospective study of 330 cases. Cytogenet Genome Res 158: 171-183, 2019.

42. Gug C, Burada F, Ioana M, Riza AL, Moldovan M, Mozos I, Rațiu A, Martiniuc V and Gorduza EV: Polyploidy in first and second trimester pregnancies in Romania. Clin Lab 66: Epub ahead of print, 2020.

This work is licensed under a Creative Commons Attribution-NonCommercial-NoDerivatives 4.0 International (CC BY-NC-ND 4.0) License. 